\title{
Electric noise spectra of a near-surface nitrogen vacancy center diamond with a protective layer
}

\author{
Philip Chrostoski*, H. R. Sadeghpour ${ }^{\dagger}$, and D. H. Santamore*,† \\ *Department of Physics and Engineering Physics, \\ Delaware State University, Dover, DE 19901, USA and \\ ${ }^{\dagger}$ ITAMP, Harvard-Smithsonian Center for Astrophysics, Cambridge, Massachusetts 02138, USA
}

(Dated: December 5, 2018)

\begin{abstract}
Surface noise is a detrimental issue for sensing devices based on shallow nitrogen vacancy (NV) color center diamonds. A recent experiment indicates that electric field noise is significant compared to magnetic field noise. They also found that the electric field noise can be reduced with a protective surface layer, though the mechanism of noise reduction is not well understood. We examine the effect of a protective surface layer on the noise spectrum, which is caused by surface charge fluctuations. We use the fluctuation-dissipation theorem to calculate and analyze the noise spectrum for six different surface layer materials typically used for NV center diamond devices. We find that four parameters largely affect the noise spectrum: effective relaxation time, effective loss tangent, power law exponent of the noise spectrum, and layer thickness. Our results suggest that a surface covering layer is indeed useful for decreasing surface noise, but which material is most suitable depends on the device operational frequency range.
\end{abstract}

\section{INTRODUCTION}

Nitrogen vacancy (NV) center diamonds are attractive candidates for a wide range of applications, ranging from quantum metrology and sensing, to quantum information processing, and hybrid quantum systems [1, 2]. Much of the interest in NV center diamonds stems from the long quantum coherence time of their spin statesseveral milliseconds at temperatures well above room temperature [3] - and their extremely high sensitivity to electric and magnetic fields[4 9]. NV color centers have a number of practical applications 10 27] in magnetic field sensing, magnetometry, scanning thermal microscopy and frequency-modulated radio reception in extreme conditions, in addition to being candidates for room temperature quantum computing.

When functioning as detectors, NV centers must be placed as close to the sample surface, for maximal detection sensitivity 28 33]. Yet room-temperature devices with NV centers near the surface tend to be noisy. This noise produces fluorescent line-broadening and decreases the overall detection sensitivity. Previously, magnetic field noise was regarded as a dominating source of noise 34 37]. The majority of magnetic field noise comes from bulk impurity interactions of nuclear and electronic spin baths [38, 39]. The surface magnetic noise also exists and has been attributed to electron spins of dangling bonds 40, 41], terminating surface atoms 42, 43], absorption of molecules 44, and interactions with paramagnetic surface molecules [45]. Magnetic surface states have been experimentally observed for both bulk and single crystalline surfaces [36, 46, 47].

However, recent experiments by Kim et al. 32 and Romach et al. 48 revealed that, in devices at room temperature, electric field fluctuations can sometimes be a larger source for noise than magnetic field fluctuations. The electric field noise may be caused by lattice strain $[9]$ and dipole fluctuations of an NV center due to interaction with fluctuating surface charges. Although, the effect of the former seems to be much smaller than that of the latter. Mamin et al. 49] were able to reduce NV center noise by placing a layer of polymethyl methacrylate atop the diamond. Kim et al. 32] also reported noise spectrum and $\mathrm{T}_{2}$ coherence time improvement with certain covering materials on NV center diamonds. Other materials, however, either reduce or increase surface noise 48, 50 52]. Until now there has been no systematic theoretical study of electric noise in NV center diamond devices with different covering layers, nor any heuristics beyond experimental intuition for determining which materials efficiently reduce noise in which frequency bands.

In this paper, we study the physics of surface electric field noise in diamonds containing NV centers. We evaluate the effect of noise reduction with a liquid or solid cover layer, and identify the parameters that control noise reduction in various situations. Since most NV center devices operate at room temperature, we treat the surface electric field noise as being produced mainly by thermally-activated fluctuating dipoles caused by the surface charges. These dipole fluctuations lead to fluctuations in the electric field at the position of the color center. We used the fluctuation-dissipation theorem for noise calculations. Kuehn et al., for example, have used the method to calculate dielectric fluctuation due to noncontact friction for PMMA [53] at a fixed frequency. We will use it to calculate frequency dependent noise with different covering materials to explore the optimal frequencies of operation for those materials.

Our theoretical model, described in Sec. III consists of a diamond coated with a cover layer, which we also sometimes refer to as a "protective layer". We use the fluctuation-dissipation theorem to obtain noise spectra at room temperature, and we calculate the effective capacitance and loss tangent. The surface cover materials we consider are: glycerol, propylene carbonate (PC), polymethyl methacrylate (PMMA), polyvinylindene flouride 
(PVDF), perflouropolyether (PFPE), and dimethyl sulfoxide (DMSO). PMMA, PVDF, PFPE, glycerol, and PC have been commonly used in experimental work 48 -52]. PMMA and PVDF are both solids, while all the others are liquid at room temperature. We analyze the noise spectra at frequencies ranging from $1 \mathrm{kHz}-10 \mathrm{MHz}$, typical in experiments, and compare the results with experimental data of Ref. [32].

We find that there are four main parameters that shape the noise spectrum: the effective relaxation time, the effective loss tangent, the coefficient of the power law of the noise spectrum, and the thickness of the surface layer. The effective capacitance also matters, but to a lesser extent. In Sec. III, we examine the role of each parameter in depth and discuss how the protective layer affects the parameters and the shape of the noise spectrum. The effective relaxation time $\tau_{\text {eff }}$ determines the transition frequency of the noise spectrum.

Our study of six experimentally representative covering materials shows that most covering materials commonly used by experimentalists can reduce noise in the frequency range of interest. Most liquid surface layers are much better than solid layers at reducing noise. This is because liquid layers have shorter (picosecondnanosecond) characteristic relaxation times than solid layers (microseconds). However, the power law exponent for liquids is in general smaller than that for solids and greatly affected by the thickness.

The frequency range where power law dominates the noise spectrum is narrower for liquids than for solids. In liquids, a constant noise floor (i.e., white noise) reappears at frequencies above $10^{5}-10^{6} \mathrm{~Hz}$, while the noise spectrum in solids still follows the power law in this frequency range. As a result, around $1 \mathrm{MHz}$, solids start to perform better in noise reduction than some liquids. At high frequencies $\left(>10^{7} \mathrm{~Hz}\right)$, a solid protective layer will outperform all liquid layers in noise reduction. The thickness of the surface layer affects the amount of noise: in general, thinner protective layers reduce white noise floor better than that of thick protective layer.

\section{MODEL AND CALCULATIONS}

\section{A. Noise spectrum}

Our model consists of a room-temperature diamond with an NV center embedded $5 \mathrm{~nm}$ below the diamond surface, with a liquid or solid layer covering the surface (see Fig. 11).

The covering materials we investigate are glycerol, PC, PMMA, PVDF, PFPE, and DMSO. The reason behind choosing these different materials is due to their favorable characteristics. For example, Kim et al. 32] has shown that glycerol does not affect the dark spin density of NV centers even though glycerol's hydroxyl groups can donate protons and possibly passivate dangling electrons. On the other hand, propylene carbonate and DMSO are

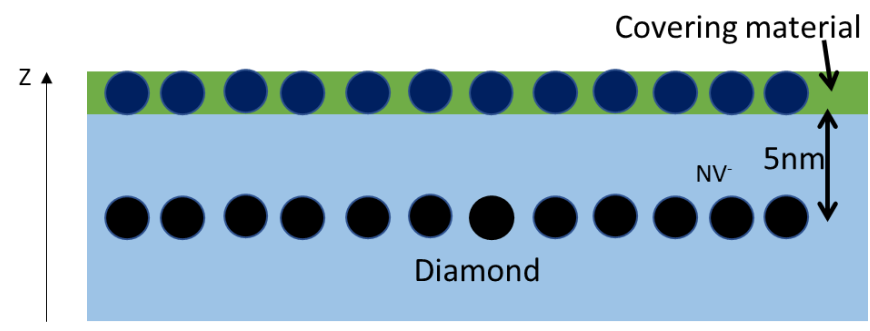

FIG. 1: The model consists of diamond with an NV center placed $5 \mathrm{~nm}$ below the surface and a protective layer covering the surface of the diamond. The lower black dots represent the NV center, while the upper blue dots represent the free positive charge of the protective material.

aprotic solvents with tightly bound atoms and are optically transparent in the wavelength regimes of NV center experiment. PMMA, PVDF, and PFPE are all nonreactive, thermally stable, and optically transparent 54 57]. PFPE has been used in space-based applications for its optical transparency, and PMMA acts like normal window glass only filtering out wavelengths below 300 $\mathrm{nm}$.

We assume that the NV centers are far apart and do not interact with each other. We focus on the noise above the NV centers and ignore the noise below them, since we are interested in noise reduction at the surface due to various protective layers. The noise from below the $\mathrm{NV}$ centers is the same for all covering layers, and so this noise source is not relevant for comparative noise reduction.

The interaction Hamiltonian of this system is

$$
H_{\mathrm{int}}=-\mathbf{p} \cdot \mathbf{E},
$$

where $\mathbf{p}$ is the electric dipole moment between the free positive charges in the covering liquid and the NV center electron and $\mathbf{E}$ is the electric field.

We want to find the noise spectrum, $S(\mathbf{k}, \omega)$, arising from the fluctuation of the dipole moment between the NV center and the covering layer. The electric field is due to charge fluctuations and acts on the system through the dipole moment. To obtain the noise spectrum, it s necessary to calculate the two-point space-time correlation of the dipole moment fluctuation, $\left\langle\delta p(\boldsymbol{r}, \tau) \delta p\left(\boldsymbol{r}^{\prime}, \tau+t\right)\right\rangle$, and then, calculate the noise spectrum by

$$
S(\mathbf{k}, \omega)=2 \int_{-\infty}^{\infty}\left\langle\delta p(\boldsymbol{r}, \tau) \delta p\left(\boldsymbol{r}^{\prime}, \tau+t\right)\right\rangle e^{-i \omega t} d t
$$

However, since the system's response function is local, fluctuations at distinct spatial coordinates are uncorrelated. Then $\delta p(\boldsymbol{r}, \tau)=\delta p(\tau)$ and $S(\mathbf{k}, \omega)=S(\omega)$. Using the fluctuation-dissipation theorem (see Appendix for details), we obtain

$$
S(\omega)=\frac{4 \hbar}{1-e^{-\hbar \omega / k_{\mathrm{B}} T}} \varepsilon_{0} \chi_{e}^{\prime \prime} .
$$


where $\chi_{e}^{\prime \prime}$ is the imaginary part of the complex electric susceptibility, $k_{\mathrm{B}}$ is the Boltzmann constant, and $T$ is the temperature.

Let $\varepsilon^{\prime}$ and $\varepsilon^{\prime \prime}$ be the real and imaginary parts of the permittivity. We rewrite $\varepsilon_{0} \chi_{e}^{\prime \prime}$ in terms of the loss tangent, $\tan \phi(\omega) \equiv \varepsilon^{\prime} / \varepsilon^{\prime \prime}$, from the complex permittivity,

$$
\varepsilon_{0} \chi_{e}^{\prime \prime}(\omega)=\varepsilon^{\prime}(\omega) \tan \phi(\omega) .
$$

We choose the axis of interaction of $\mathbf{p}$ to be the $z$-axis as shown in Fig. 1 and model the two layers above the NV center - the diamond and cover layers - each with capacitance $C$. The system is analogous to parallel-plate capacitors in series due to the intrinsic electric field naturally generated by the dipole interactions between the negative charge in the NV center and the positive charge in the covering layer. After some manipulation, the noise spectrum per unit volume becomes

$$
\bar{S}(\omega)=\frac{4 \hbar C(\omega)}{1-e^{-\frac{\hbar \omega}{k_{B} T}}} \tan \phi(\omega) .
$$

\section{B. Effective capacitance and effective loss tangent}

Equation 4 contains the capacitance $C(\omega)$ and loss tangent, $\tan \phi(\omega)$. The capacitance expressed in terms of the electric field as $C(\omega)=1 / E(\omega) d$, with

$$
E(\omega)=\frac{\kappa q}{\varepsilon^{\prime}(\omega) d^{2}},
$$

where $\kappa=1 / 4 \pi \varepsilon_{0}, q$ is the charge, and $d$ is the thickness of the layer. This expression lets us write capacitance in terms of electric permittivity. The frequencydependent permittivity for a single material is a relaxation permittivity given by the Havriliak-Negami relaxation function [58]

$$
\varepsilon(\omega)=\varepsilon_{\infty}+\frac{\triangle \varepsilon}{\left(1+(i \omega \tau)^{\gamma}\right)^{\beta}},
$$

where $\triangle \varepsilon=\varepsilon_{\infty}-\varepsilon_{s}$ is the difference between the limiting high-frequency permittivity $\varepsilon_{\infty}$ and the limiting lowfrequency "static" permittivity $\varepsilon_{s}$, and $\tau$ is the medium's characteristic relaxation time, given in terms of the material's relaxation frequency $f_{r}$ by $\tau=1 / 2 \pi f_{r}$. The exponents $\gamma$ and $\beta$ are fractional shape parameters describing the skewing and broadening of the dielectric function.

For a diamond without any protective layer, the capacitance $C(\omega)$ and loss tangent $\tan \phi(\omega)$ in Eq. (4) are simply calculated from the properties of diamond. However, with a protective layer on the surface, we need to compute and use an effective capacitance $C_{\text {eff }}(\omega)$ and loss tangent $\tan \phi_{\text {eff }}(\omega)$ to calculate $\bar{S}(\omega)$. We model the structure as a "covering layer" above the top diamond layer (i.e., the $5 \mathrm{~nm}$ diamond layer directly above the NV center). Then, the effective loss tangent $\tan \phi_{\text {eff }}(\omega)$ is given by [59 62],

$$
\tan \phi_{\mathrm{eff}}(\omega)=b_{H} d_{H} \tan \phi_{H}(\omega)+b_{L} d_{L} \tan \phi_{L}(\omega),
$$

where $H$ and $L$ respectively denote the higher and lower index of refraction of the surface liquid and the top diamond layer. The coefficients $d_{L}$ and $d_{H}$ are the two materials' thicknesses, and

$$
b_{L, H}=\frac{1}{\sqrt{\pi} w}\left(\frac{Y_{L, H}}{Y_{s}}+\frac{Y_{s}}{Y_{L, H}}\right)
$$

where $w$ is the width and $Y_{L, H, s}$ is the Young's modulus. The subscript $s$ refers to the substrate, that is, the diamond below the NV center. We have written this formula as $Y_{L, H, s}$ even though $s=L$ or $s=H$-either the $L$ layer or the $H$ layer is diamond, as is the substrate so that Eq. (8) can be used for two different covering layers on the diamond surface if needed. The effective capacitance $C_{\text {eff }}(\omega)$ is the capacitance of the two layers of dielectric materials, equivalently to the diamond and the protective layer in series. The material parameters used in our calculations are taken from the literature 63 . and are shown in Table \.

\begin{tabular}{|l|l|l|l|l|l|l|}
\hline material & $\varepsilon_{s}$ & $\varepsilon_{\infty}$ & $\mathrm{Y}(\mathrm{GPa})$ & $\tau(\mathrm{ns})$ & $\gamma$ & $\beta$ \\
\hline diamond & 7.99 & 5.7 & 443 & 2480 & 0.97 & 0.89 \\
\hline glycerin & 51.8 & 3.9 & 4.80 & 19.9 & 1.00 & 0.67 \\
\hline PC & 64.9 & 4.7 & 8.09 & 0.0433 & 0.985 & 0.927 \\
\hline DMSO & 45.9 & 6.36 & 4.50 & 11.0 & 1.00 & 1.00 \\
\hline PMMA & 3.60 & 2.6 & 5.00 & 500 & 1.00 & 1.00 \\
\hline PVDF & 7.50 & 5.0 & 1.10 & 100000 & 1.00 & 1.00 \\
\hline PFPE & 104 & 5.0 & 34.5 & 7.00 & 0.87 & 1.00 \\
\hline
\end{tabular}

TABLE I: Material parameters used in Eq. (8) and Eq. (6)

\section{RESULTS AND DISCUSSION}

Fig. 2 shows calculated noise spectra as a function of frequency for bare diamonds and for diamonds with various surface protective layers of thickness $5 \mathrm{~nm}$.

At low frequencies the noise spectra exhibit white noise, with a transition at higher frequencies to a power law spectrum, with $S(\omega) \propto 1 / f^{a}$. This agrees with the experimental findings of Romach et al. 48]. White noise at low frequencies comes about when dipoles try to align themselves into an equilibrium state.

We see from Fig. 2 that all surface covering materials we examined reduce the noise in some though not all frequency ranges. This agrees with the experimental observations reported for glycerin and propylene carbonate by Kim, et al. [32]. However, certain materials are more effective in reducing noise at low frequencies, while others work better at high frequencies. For example, PVDF generates more noise than bare diamond at frequencies less than $3 \times 10^{4} \mathrm{~Hz}$ but surpasses PMMA in noise reduction above $2 \times 10^{5} \mathrm{~Hz}$, glycerol above $4 \times 10^{5} \mathrm{~Hz}$, and PFPE above $10^{6} \mathrm{~Hz}$. 


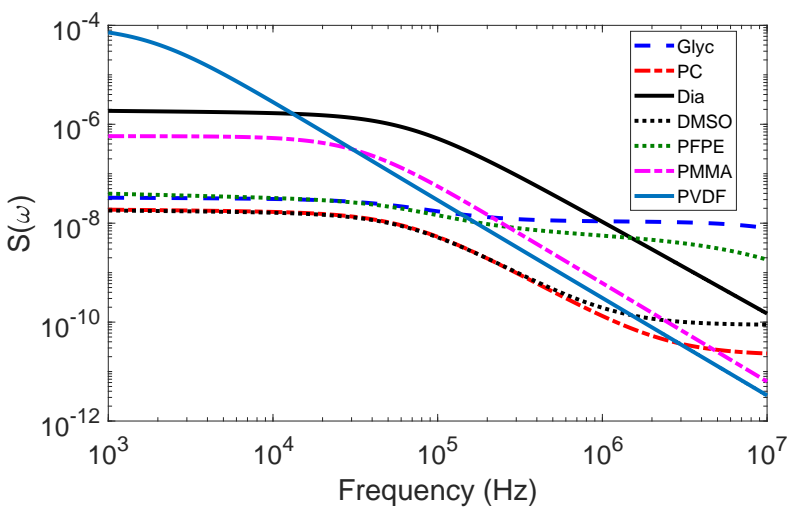

FIG. 2: Charge noise spectrum: bare diamond (black); with glycerol (blue dashed); propylene carbonate (red dashed); dimethyl sulfoxide (black dotted); perfluoropolyether (green dotted); polyvinylidene fluoride (navy); and polymethyl methacrylate (pink dashed). The NV center diamond with a liquid protective layer exhibits better noise reduction than the bare diamond and the diamond with a solid protective layer in the frequency range $<10^{6} \mathrm{~Hz}$.

Four parameters largely affect the shape of noise spectrum at room temperature: the effective relaxation time $\tau_{\text {eff }}$, the effective loss tangent $\tan \phi_{\text {eff }}(\omega)$, the power law coefficient $a$, and the thickness of the surface layer. The effective capacitance also affects the noise spectrum; however, the effect is minimal compared to the other parameters, because of very little variation of the real permittivity $\epsilon^{\prime}$ with frequency (see Sec. IIIB).

\section{A. Effective relaxation time}

The effective relaxation time $\tau_{\text {eff }}$ determines the transition frequency $f_{t}$ at which the spectrum transitions from white noise to a power law. The relationship is $f_{t}=2 \pi / \tau_{\text {eff }}$, where $1 / \tau_{\text {eff }}=1 / \tau_{\text {dia }}+1 / \tau_{\text {surf }}$. Here $\tau_{\text {dia }}$ and $\tau_{\text {surf }}$ are the material relaxation times of the diamond and the protective surface layer, respectively. Thus, for a liquid surface protective layer, in which $\tau_{\text {surf }} \gg \tau_{\text {dia }}$, the effective transition time is dominantly determined by the relaxation time of the diamond. With a solid surface, on the other hand, $\tau_{\text {surf }}$ is comparable to $\tau_{\text {dia }}$, and thus the effective relaxation time will be determined by both $\tau_{\text {surf }}$ and $\tau_{\text {dia }}$. We discuss the effect of thickness in Sec. IIID.

\section{B. Effective loss tangent}

Figures 2 and 3(a) show that the amount of white noise is determined by the effective loss tangent $\tan \phi_{\text {eff }}(\omega)$. In addition, overall charge fluctuation noise is proportional to the dielectric loss tangent, and thus charge fluctuation noise increases for lossy materials.

Next, since loss tangent is defined as the ratio of the imaginary permittivity $\epsilon^{\prime \prime}$ to the real permittivity $\epsilon^{\prime}$, we

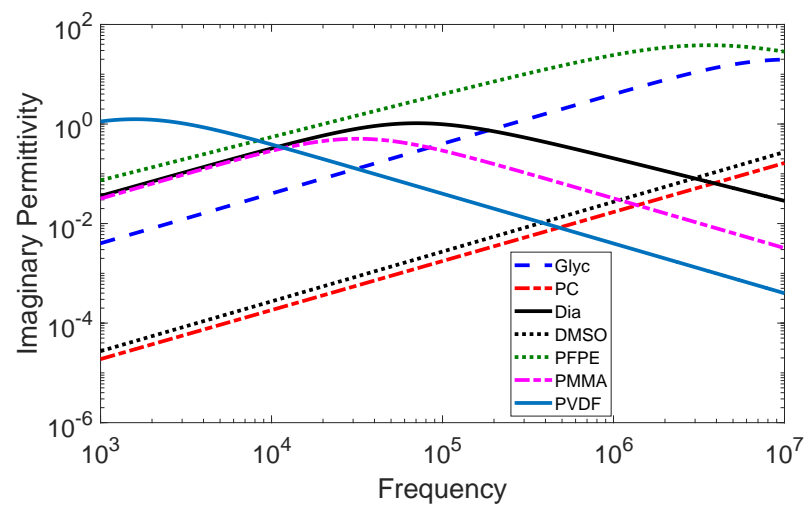

(a) Imaginary permittivity.

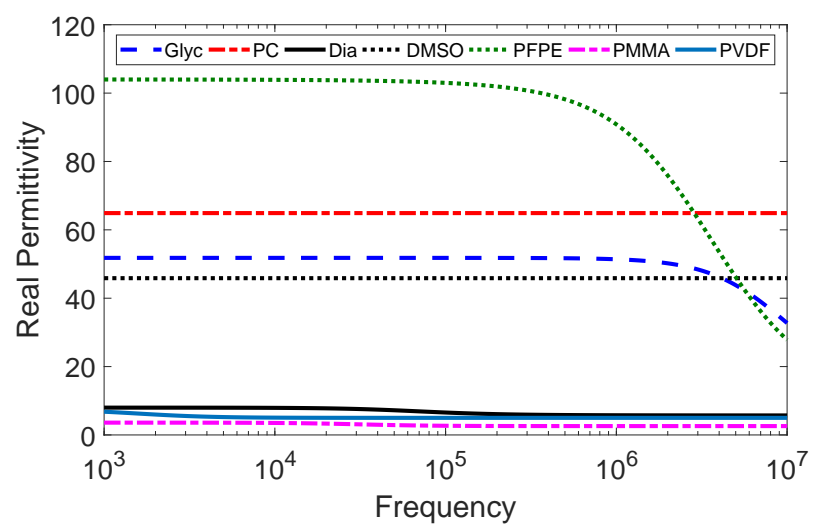

(b) Real permittivity. The real part of the permittivity is almost constant with frequency except at frequencies around $1 / \tau$.

FIG. 3: Imaginary and real permittivities as a function of frequency: bare NV center diamond (black); with glycerol (blue dashed); propylene carbonate (red dashed); dimethyl sulfoxide (black dotted); perfluoropolyether (green dotted); polyvinylidene fluoride (navy); and polymethyl methacrylate (pink dashed)

look into both $\epsilon^{\prime}$ and $\epsilon^{\prime \prime}$. Compared to the imaginary part (see Fig. 3(a)), the real part of the permittivity is relatively constant with frequency throughout the frequency range $10^{3}-10^{7} \mathrm{~Hz}$, except around the transition frequency $f_{t}$ (see Fig. 3(b)). As a result, $\tan \phi_{\text {eff }}(\omega)$ is predominantly determined by the imaginary permittivity. This could be why Kim et al. [32], who used only real permittivity to estimate the noise, could not explain the noise spectra they observed in their experiments.

The noise spectrum is also affected by the capacitance, as shown in Eq. (4), and thus it is also proportional to the real part of the permittivity. However, since the real permittivity is relatively constant with frequency, except around the transition frequency $f_{t}$, the effect of capacitance on the noise spectrum is much less than that of the loss tangent. 


\section{Power law exponent}

In atomic systems such as an ion trap, the power law $1 / f^{a}$ is associated with the number of excitation modes of environmental phonons [64]. However, in our study, temperature is held constant, and thus the excitation modes of environmental phonons are the same for all materials. As can be read off Fig. 2, the noise spectra of the solid systems we considered (bare diamond, PVDF+diamond, and PMMA+diamond) follow a power law with exponent ranging from $a=-2.1$ to $a=-2$. On the other hand, with a liquid surface layer such as glycerin, PC, DMSO, and PFPE, the power law exponent varies from $a=-0.5$ to $a=-1.9$. The exponent depends on thickness, as discussed in the next subsection IIID.

\section{Thickness of the surface layer}

\begin{tabular}{|l|l|l|}
\hline material & $a(d=5 \mathrm{~nm})$ & $a(d=1 \mu \mathrm{m})$ \\
\hline PC & -1.6 & -0.91 \\
\hline glycerin & -0.5 & -0.15 \\
\hline Silicon & -1.89 & -1.92 \\
\hline DMSO & -1.9 & -1.1 \\
\hline PFPE & -1.6 & -0.2 \\
\hline PMMA & -2.2 & -2.1 \\
\hline PVDF & -2.0 & -1.94 \\
\hline
\end{tabular}

TABLE II: Noise power exponent of seven materials examined with thickness $1 \mathrm{~nm}$ and $1 \mu \mathrm{m}$.

Table II shows that for liquid surface layers, the power law exponent $a$ varies drastically with thickness. For solid surface layers, though, a does not vary much with thickness. The power law region occurs when the dipole moments of the surface covering layer have aligned themselves to the NV center. Thus, the power law of a liquid covered diamond seems to be strongly affected by the stability of aligned dipole moments than that of a solid covered diamond.

Figure 4 shows the noise spectra with thick $(1 \mu \mathrm{m})$ surface protective layers. The thickness of the protective layer does not change the noise spectrum transition frequency $f_{t}$, since the transition is governed by the relaxation time. However, a thick surface protective layer does increase the noise floor in general.

The exception to this rule is for those materials with a very short relaxation time $\tau_{\text {surf }}$, such as PC and DMSO and only at low frequency range (noise floor is high for high frequency range). This puzzling phenomenon can be explained when we re-express $\bar{S}(\omega)$ in Eq. (4) by explicitly writing the effective capacitance in terms of geometry and factoring out thickness independent terms.

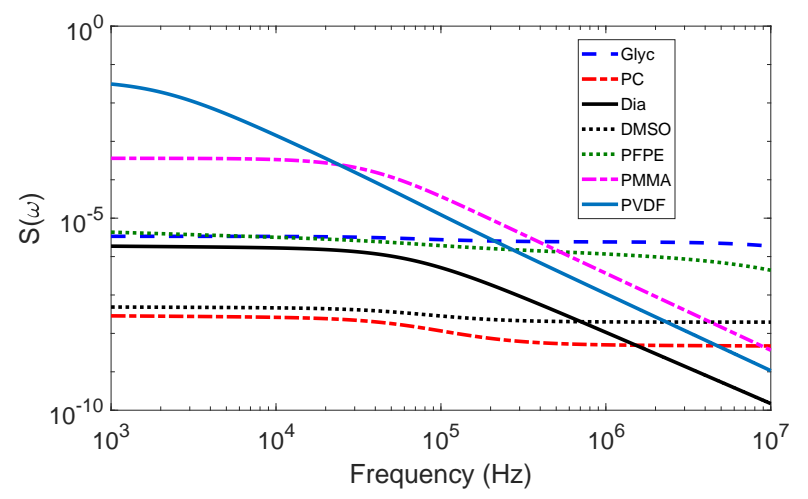

FIG. 4: Noise spectrum with thickness $1 \mu \mathrm{m}$. Thicker surface layers have higher noise floor. The power law exponent changes with thickness for liquid surface layers but almost the same for solid surface layers.

After some simplification, we obtain

$$
\bar{S}(\omega) \approx \Gamma\left(\frac{b_{L}}{\varepsilon_{H}^{\prime}} \frac{d_{L}}{d_{H}} \phi_{L}+\frac{b_{H}}{\varepsilon_{H}^{\prime}} \phi_{H}\right) .
$$

where $\Gamma$ incorporates all factors independent of layer thickness. The first term of Eq. (9) contains the ratio of the thicknesses of the protective layer and top diamond layer; it is the only thickness dependent part of the equation. Note, however, that this first term also depends on the loss tangent. As a result, in materials such as PC and DMSO whose loss tangent $\phi_{L}$ is much smaller at low frequencies than that of diamond, the first term will be negligible compared to the second term. Thus, in these materials, thickness makes almost no difference to the noise spectrum at low frequencies as seen in Fig. 4 .

\section{E. Which cover layers reduce noise most effectively?}

Having highlighted the roles of four parametersrelaxation time, loss tangent, power law, and thicknessin noise reduction, we now draw out some practical implications of our results. At low frequencies, fluctuations in dipole-dipole interactions manifests as white noise. Because its characteristic relaxation time is shorter than that of diamond, a thin $(<10 \mathrm{~nm})$ protective layer can reduce noise by decreasing the effective loss tangent. In the frequency range understudy, liquid surface layers reduce noise more effectively than solid surface layers because the picosecond-to-nanosecond relaxation times of liquids is much shorter than the microsecond relaxation times of solids. Because they can move more easily than in solids, the dipoles in liquids realign more quickly than the dipoles in solids. The low mobility in solids and the consequently slower realignment of dipoles leads to more fluctuation noise. On the other hand, the power law coefficient in liquids is generally smaller than in solids, and the frequency range over which the power law holds is 
narrower in liquids than in solids. In liquids, the noise spectrum becomes flat again at frequencies beyond a few megahertz $\left(10^{6} \mathrm{~Hz}\right)$, whereas in solids the power law persists even at higher frequencies. As a result, at high frequencies $\left(>10^{7} \mathrm{~Hz}\right)$, solid protective layers outperform liquid protective layer in noise reduction. Furthermore, the thickness of the surface layer also affects the amount of noise: the thinner the protective layer, the better the noise reduction. A solid protective surface thicker than $10 \mathrm{~nm}$ may increase noise rather than reduce it.

\section{CONCLUSIONS}

We have studied the effectiveness at reducing surface noise of protecting layers on NV center diamonds. We have assumed that the main source of the noise is thermally activated surface charge fluctuations. This assumption is reasonable, since the NV center based devices in question operate at room temperature, and various experiments indicate that the noise mainly originates from the electric field 32,48$]$. We have used the quantum fluctuation-dissipation theorem to calculate the noise spectrum. We have analyzed the noise spectra for six materials commonly used to cover the surface of NV center diamonds: glycerol, propylene carbonate, polymethyl methacrylate, polyvinylindene flouride, perflouropolyether, and dimethyl sulfoxide. The covering materials examined in this work all exhibit optical transparency in NV center experiments and do not interfere with NV center readout [54 57]. While chemical reactivity with biomolecules for sensing could possibly be affected by the coating, reduction in noise may more than compensate for this effect.

Our results show that four parameters affect the noise spectra: the effective relaxation time, the effective loss tangent, the exponent of the power law, and the thickness of the surface layer. (The effective capacitance also influences the overall noise spectra, but to a much smaller extent.) Of these four parameters, the effective relaxation time, the effective loss tangent, and the coefficient of the power law determine the shape of the noise spectra, while the thickness of the surface layer determines the overall amount of noise. The effective relaxation time $\tau_{\text {eff }}$ determines the transition frequency of the noise spectrum: the longer the relaxation time, the lower the transition frequency. The power law behavior is associated with the stability of aligned dipole moments. Thus solid cover materials have higher power law exponents (around -2) that are very little influenced by the thickness, while liquid cover materials exhibit a drastic decrease in the power law coefficient as the thickness increases. Consequently, one can control the power law behavior by adjusting the cover layer thickness, if liquid covering layer is used. The amount of white noise is determined by a combination of the effective loss tangent and thickness. In addition, overall charge fluctuation noise is proportional to the loss tangent, and thus the surface noise is worse for so-called "lossy materials". In general, the noise floor considerably increases with increasing thickness. The only exception to this rule is a material with very short relaxation time, such as PC and DMSO, and only at low frequency ranges. The thickness of the surface layer does not affect the transition frequency from the white noise to a power law of the noise spectrum.

Our study of six experimentally representative covering materials shows that most covering materials commonly used by experimentalists can reduce noise in the frequency range of $10^{3}-10^{7} \mathrm{~Hz}$. Liquid surface layers reduce noise better overall than solid layers, because their relaxation times are shorter. The ideal material for reducing NV center surface noise would be a thin liquid layer with a low real permittivity and a fast (picosecond range) relaxation time in the frequency range $10^{3}$ $10^{7} \mathrm{~Hz}$. Solid materials in general are noisier, but their high power law exponents compensates for the substantial white noise and provides better noise reduction at high frequencies $\left(>10^{7} \mathrm{~Hz}\right)$.

It should be noted that PMMA is shown to enhance emission that might result in reduction in lifetime 69. However, due to the complexity of the nano-diamond tip structure, such measurements may not be directly applicable to our case: in our case, the NV center is embedded in the bulk diamond.

Finally, the electronic field noise will decrease $T_{2}$ coherence time just as magnetic field noise does. Since some experiments such as 9, 32] show that the electric field noise and magnetic field noise are comparable, reducing the electric field noise might extend $T_{2}$ time considerably.

\section{Acknowledgments}

We thank Pauli Kehayias and others in Ron Walsworth group at Harvard University for useful discussions and providing experimental insights. DHS is grateful to ITAMP for hosting her visit during the summer and providing a stimulating research environment. This work is supported by the NSF grant DMR-1505641.

\section{Appendix A: Noise spectrum derivation}

In this appendix, we derive the noise spectrum via the fluctuation-dissipation theorem. We treat the the dipoledipole interaction Hamiltonian between liquid and the NV center as perturbation

$$
\delta H=-\mathbf{p} \cdot \mathbf{E}
$$

where $\mathbf{p}$ is the electric dipole moment between the charges in the covering liquid and the NV center, and $\mathbf{E}$ is the electric field. We treat the interaction using the electric dipole approximation. At thermal equilibrium, the ensemble average of the dipole moment is defined as, 


$$
\langle\mathbf{p}\rangle \equiv\langle\mathbf{p}(\mathbf{r}, t)\rangle=\frac{\int f_{\text {eq }}(\mathbf{r}) \mathbf{p}(\mathbf{r}, t) d r}{\int f_{\text {eq }}(\mathbf{r}) d r}
$$

where $f_{\text {eq }}$ is the distribution function

$$
f(x)=f_{\mathrm{eq}} e^{-\left(\delta H / k_{\mathrm{B}} T\right)} .
$$

where $k_{\mathrm{B}}$ is the Boltzmann constant and $T$ is the temperature, and the integration runs over all coordinates because the system is at equilibrium and $t$ is time. The fluctuation is regarded as a system that has been perturbed by $\delta \mathbf{p}$

$$
\delta \mathbf{p}=\mathbf{p}-\langle\mathbf{p}\rangle .
$$

This perturbation is small and linearly dependent on $\mathbf{E}$. This dependence can be given by linear response function theory [65 67],

$$
\delta p_{j}(t)=\frac{1}{2 \pi} \sum_{k} \int_{-\infty}^{t} \tilde{\alpha}_{j k}\left(t-t^{\prime}\right) E_{k}\left(t^{\prime}\right) d t^{\prime} .
$$

Here $\tilde{\alpha}_{j k}$ is the response function of the system and $j, k=$ $x, y, z$. We have assumed that the system is stationary (i.e., the response function is local) and the dependence on $t-t^{\prime}$ enforces causality. The "memory" of the system is contained in $\tilde{\alpha}_{j k}$ and we need to determine $\tilde{\alpha}_{j k}$.

Time-dependence of the electric field perturbation is a step function to ensure complete relaxation of the system at times $t=0$, and the perturbation occurs $[0, \infty)$ with $E=E_{\mathrm{k}}^{0}$ at $t>0$. Evaluating Eq. (A5) for the step function perturbation we get,

$$
\delta p_{j}(t)=\frac{E_{k}^{0}}{2 \pi} \int_{t}^{\infty} \tilde{\alpha}_{j k}(\tau) d \tau,
$$

where $\tau \equiv t-t^{\prime}$. Solving for $\tilde{\alpha}_{j k}$ we obtain,

$$
\tilde{\alpha}_{j k}(t)=-\frac{2 \pi}{E_{k}^{0}} \Theta(t) \frac{d}{d t} \delta p_{j}(t) .
$$

We assumed that $\tilde{\alpha}_{j k}(t)$ and its time-derivative tends to zero as $t \rightarrow \infty$ and $\Theta(t)$ is the Heaviside step function to ensure causality. Since the ensemble average is independent of time,

$$
\tilde{\alpha}_{j k}(t)=-\frac{2 \pi}{E_{k}^{0}} \Theta(t) .
$$

At time $t=0$, the system is in thermal equilibrium. Expanding the exponential in a series and plugging into
Eq. A22, and keeping only the terms up to linear order in $\delta H$ we obtain,

$$
\mathbf{p}=\langle\mathbf{p}\rangle-\frac{1}{k_{B} T}[\langle\delta H(s) \mathbf{p}(s, t)\rangle-\langle\mathbf{p}(s, t)\rangle\langle\delta H(s)\rangle] .
$$

Since $\delta H(s)$ is the perturbation at time $t=0$ we have $\delta H(s)=-\mathbf{p}(s, 0) E_{\mathbf{k}}^{0}$ and Eq. (A99) becomes,

$$
\delta p(t)=\frac{E_{\mathbf{k}}^{0}}{k_{B} T}\left\langle\delta p_{k}(0) \delta p_{j}(t)\right\rangle .
$$

Inserting this result into Eq. (A7) we find,

$$
\tilde{\alpha}_{j k}(t)=\frac{2 \pi}{k_{\mathrm{B}} T} \Theta(t) \frac{d}{d t}\left\langle\delta p_{k}(0) \delta p_{j}(t)\right\rangle .
$$

It is convenient to do a Fourier transform to express Eq. (A11) in the frequency domain with $\tilde{\alpha}_{j k}(t) \rightarrow$ $\alpha_{j k}(\omega)$ and $\delta p(t) \rightarrow \delta \tilde{p}_{j}(\omega)$. The correlation function in frequency domain, $\left\langle\delta \tilde{p}_{j}(\omega) \delta \tilde{p}_{k}^{*}\left(\omega^{\prime}\right)\right\rangle$ is

$$
\begin{aligned}
& \left\langle\delta \tilde{p}_{j}(\omega) \delta \tilde{p}_{k}^{*}\left(\omega^{\prime}\right)\right\rangle \\
& =\delta\left(\omega-\omega^{\prime}\right) \frac{1}{2 \pi} \int_{-\infty}^{\infty}\left\langle\delta \tilde{p}_{k}(t) \delta \tilde{p}_{j}^{*}(t+\tau)\right\rangle e^{i \omega t} d t .
\end{aligned}
$$

To obtain a spectral representation of the fluctuationdissipation theorem, we need to Fourier transform Eq. (A11). The right hand-side leads to a convolution between the spectrum of the step function $\Theta(t)$ and the spectrum of $d / d t\left\langle\delta p_{k}(0) \delta p_{j}(t)\right\rangle$. To remove of the imaginary part of the step function, we solve for $\left[\alpha_{j k}(\omega)-\alpha_{k j}^{*}(\omega)\right]$ instead of $\alpha_{j k}(\omega)$. Using the WienerKhintchine theorem [68] and taking the real part (note that $\left\langle\delta p_{k}(t) \delta p_{j}(t+\tau)\right\rangle$ is real) we get

$$
\left\langle\delta \tilde{p}_{j}(\omega) \delta \tilde{p}_{k}^{*}\left(\omega^{\prime}\right)\right\rangle=\frac{k_{B} T}{2 \pi i \omega}\left[\alpha_{j k}(\omega)-\alpha_{k j}^{*}(\omega)\right] \delta\left(\omega-\omega^{\prime}\right) .
$$

The Wiener-Khintchine theorem applies to classical systems, where $\hbar \omega \ll k_{\mathrm{B}} T$. However, we can generalize it to quantum systems by replacing $k_{B} T$ by $\hbar \omega /\left(1-e^{-\hbar \omega / k_{B} T}\right)$ and substituting into Eq. (A13). Then $\left\langle\delta \tilde{p}_{j}(\omega) \delta \tilde{p}_{k}^{*}\left(\omega^{\prime}\right)\right\rangle$ becomes

$$
\begin{aligned}
\left\langle\delta \tilde{p}_{j}(\omega) \delta \tilde{p}_{k}^{*}\left(\omega^{\prime}\right)\right\rangle & =\frac{1}{2 \pi i \omega}\left[\frac{\hbar \omega}{1-e^{-\hbar \omega / k_{B} T}}\right] \\
& \times\left[\alpha_{j k}(\omega)-\alpha_{k j}^{*}(\omega)\right] \delta\left(\omega-\omega^{\prime}\right) .
\end{aligned}
$$

Fluctuation is now converted to dissipation on the right hand side. We now need to determine $\alpha_{j k}(\omega)-\alpha_{k j}^{*}(\omega)$. Dissipation in the system is associated with the imaginary part of the complex electric susceptibility, $\chi_{e}^{\prime \prime}$, since 
$p \propto \varepsilon_{0} \chi_{e}$, where $\varepsilon_{0}$ is the vacuum permittivity and $\chi_{e}$ is the complex electric susceptibility. Therefore, the dissipation term is contained in $\chi_{e}^{\prime \prime}$, and $\alpha_{j k}(\omega)$ is thus $i \varepsilon_{0} \chi_{e}^{\prime \prime}$.

The noise spectrum is obtained from the fluctuation of the dipole moment by plugging $\alpha_{j k}(\omega)=i \varepsilon_{0} \chi_{e}^{\prime \prime}$ in Eq. (A14) and then into Eq. (2)

$$
S(\mathbf{k}, \omega)=2 \int_{-\infty}^{\infty}\left\langle\delta p(\boldsymbol{r}, \tau) \delta p\left(\boldsymbol{r}^{\prime}, \tau+t\right)\right\rangle e^{-i \omega t} d t
$$

However, since the system's response function is local, fluctuations at distinct spatial coordinates are uncorrelated. Then $\delta p(\boldsymbol{r}, \tau)=\delta p(\tau)$ and $S(\mathbf{k}, \omega)=S(\omega)$, so that

$$
S(\omega)=\frac{4 \hbar}{1-e^{-\hbar \omega / k_{B} T}} \varepsilon_{0} \chi_{e}^{\prime \prime} .
$$

[1] L. Childress, R. Walsworth, and M. Lukin, Atom-like crystal defects: From quantum computers to biological sensors, Physics Today 67 (10), 38 (2014).

[2] Z. Xiang, S. Ashhab, J. Q. You, F. Nori, Hybrid quantum circuits: Superconducting circuits interacting with other quantum systems, Rev. Mod. Phys. 86, 623 (2013).

[3] G. Balasubramanian, P. Neumann, D. Twitchen, M. Markham, R. Kolesov, N. Mitzuochi, J. Isoya, J. Achard, J. Beck, J. Tissler, V. Jacques, P. R. Hemmer, F. Jelezko, and J. Wrachtrup, Ultralong spin coherence time in isotopically engineered diamond, Nat. Mater. 8, 383 (2009).

[4] G. Balasubramanian, I. Y. Chan, R. Kolesov, M. AlHmoud, J. Tissler, C. Shin, C. Kim, A. Wojcik, P. R. Hemmer, A. Krueger, T. Hanke, A. Leitenstorfer, R. Bratschitsch, F. Jelezko, and J. Wrachtrup, Nanoscale imaging magnetometry with diamond spins under ambient conditions, Nature 455, 648-651 (2008).

[5] J. R. Maze, P. L. Stanwix, J.S. Hodges, S. Hong, J. M. Taylor, P. Cappellaro, L. Jiang, M. V. Gurudev Dutt, E. Togan, A. S. Zibrov, A. Yacoby, R. L. Walsworth, and M. D. Lukin, Nanoscale magnetic sensing with an individual electronic spin in diamond", Nature 455, 644-647 (2008).

[6] M. S. Grinolds, S. Hong, P. Malentinsky, L. Luan, M. D. Lukin, R. L. Walsworth, and A. Yacoby, "Nanoscale magnetic imaging of a single electron spin under ambient conditions, Nature Physics 9, 215-219 (2013).

[7] E. Togan, Y. Chu, A. S. Trifonov, L. Liang, J. Maze, L. Childress, M. V. G. Dutt, A. S. Sorensen, P. R. Hemmer, A. S. Zibrov, and M. D. Lukin, Quantum entanglement between an optical photon and a solid-state spin qubit, Nature 466, 730-734 (2010).

[8] G. Waldherr, P. Neumann, S. F. Huelga, F. Jelezko, and J. Wrachtrup, Violation of a Temporal Bell Inequality for Single Spins in a Diamond Defect Center, Phys. Rev. Lett. 107, 090401 (2011).

[9] B. A. Myers, A. Ariyaratne, and A. C. Bleszynski Jayich, Double-Quantum Spin-Relaxation Limits to Coherence of Near-Surface Nitrogen-Vacancy Centers, Phys. Rev. Lett. 118, 197201 (2017).

[10] K. D. Jahnke, B. Naydenov, T. Teraji, S. Koizumi, T. Umeda, J. Isoya, and F. Jelezko, Long coherence time of spin qubits in ${ }^{12} \mathrm{C}$ enriched polycrystalline chemical vapor deposition diamond, Appl. Phys. Lett. 101, 012405 (2012).

[11] M. W. Doherty, V. M. Acosta, A. Jarmola, M. S. J. Barson, N. B. Manson, D. Budker, and L. C. L. Hollenberg, Temperature shifts of the resonances of the NV center in diamond, Phys. Rev. B 90, 041201(R) (2014).

[12] A. Laraoui, F. Dolde, C. Burk, F. Reinhard, J. Wrachtrup, and C. A. Meriles, High-resolution correlation spectroscopy of ${ }^{13} \mathrm{C}$ spins near a nitrogen-vacancy centre in diamond, Nat. Commun. 4, 1651 (2013).

[13] J. M. Taylor, P. Cappelaro, L. Childress, L. Jiang, D. Budker, P. R. Hemmer, A. Yacoby, R. Walsworth, and M. D. Lukin, High-sensitivity diamond magnetometer with nanoscale resolution, Nat. Phys. 4, 810 (2008).

[14] C. L. Degan, Scanning magnetic field microscope with a diamond single-spin sensor, Appl. Phys. Lett, 92, 243111 (2008).

[15] V. M. Acosta, E. Bauch, M. P. Ledbetter, C. Santori, K. M. C. Fu, P. E. Barclay, R. G. Beausoleil, H. Linget, J. F. Roch, F. Treussart, S. Chemerisov, W. Gawlik, and D. Budker, Diamonds with a high density of nitrogenvacancy centers for magnetometry applications, Phys. Rev. B 80, 115202 (2009).

[16] A. Jarmola, Z. Bodrog, P. Kehayias, M. Markham, J. Hall, D. J. Twitchen, V. M. Acosta, A. Gali, and D. Budker, Optically detected magnetic resonances of nitrogenvacancy ensembles in $13 \mathrm{C}$-enriched diamond, Phys. Rev. B. 94, 094108 (2016).

[17] F. Dolde, H. Fedder, M. W. Doherty, T. Nöbauer, F. Rempp, G. Balasubramanian, T. Wolf, F. Reinhard, L. C. Hollenberg, F. Jelezko, and J. Wrachtrup, Electricfield sensing using single diamond spins, Nature Physics 7, 459 (2011).

[18] M. W. Doherty, F. Dolde, H. Fedder, F. Jelezko, J. Wrachtrup, N. B. Manson, and L. C. L. Hollenberg, Theory of the ground-state spin of the $\mathrm{NV}^{-}$center in diamond, Phys. Rev. B 85, 205203 (2012)

[19] F. Dolde, M. W. Doherty, J. Michl, I. Jakobi, B. Naydenov, S. Pezzagna, J. Meijer, P. Neumann, F. Jelezko, N. B. Manson, and J. Wrachtrup, Nanoscale Detection of a Single Fundamental Charge in Ambient Conditions Using the $\mathrm{NV}^{-}$Center in Diamond", Phys. Rev. Lett. 112, 097603 (2014).

[20] P. Ovartchaiyapong, K. W. Lee, B. A. Myers, and A. C. B. Jayich, Dynamic strain-mediated coupling of a single diamond spin to a mechanical resonator", Nat. Commun. 5, 4429 (2014).

[21] E. R. MacQuarrie, T. A. Gosavi, N. R. Jungwirth, S. A. Bhave, and G. D. Fuchs, Mechanical Spin Control of Nitrogen-Vacancy Centers in Diamond, Phys. Rev. Lett. 111, 227602 (2013).

[22] D. M. Toyli, C. F. de las Casas, D. J. Christle, V. V. Dobrovitski, and D. D. Awschalom, Fluorescence thermometry enhanced by the quantum coherence of single spins in diamond, Proc. Natl. Acad. Sci. U.S.A. 110, 8417 (2013).

[23] G. Kucsko, P. C. Maurer, N. Y. Yao, M. Kubo, H. J. Noh, P. K. Lo, H. Park, and M. D. Lukin, Nanometrescale thermometry in a living cell, Nature 500, 54 (2013).

[24] P. Neumann, N. Mizouchi, F. Rempp, P. Hemmer, H. 
Watanabe, S. Yamasaki, V. Jacques, T. Gaebel, F. Jelezko, and J. Wrachtrup, Multipartite Entanglement Among Single Spins in Diamond, Science 320, 1326 (2008).

[25] P. Neumann, R. Kolesov, B. Naydenov, J. Beck, F. Rempp, M. Steiner, V. Jacques, G. Balasubramanian, M. L. Markham, D. J. Twitchen, S. Pezzagna, J. Meijer, J.Twamley, F. Jelezko, and J. Wrachtrup, Quantum register based on coupled electron spins in a roomtemperature solid, Nat. Phys. 6, 249 (2010).

[26] P. Neumann, J. Beck, M. Steiner, F. Rempp, H. Fedder, P. R. Hemmer, J. Wrachtrup, and F. Jelezko, SingleShot Readout of a Single Nuclear Spin, Science 329, 542 (2010).

[27] G. Waldherr, J. Beck, M. Steiner, P. Neumann, A. Gali, Th. Frauenheim, F. Jelezko, and J. Wrachtrup, Dark States of Single Nitrogen-Vacancy Centers in Diamond Unraveled by Single Shot NMR, Phys. Rev. Lett. 106, 157601 (2011).

[28] T. Staudacher, F. Shi, S. Pezzagna, J. Meijer, J. Du, C. A. Meriles, F. Reinhard, J. Wrachtrup, Nuclear Magnetic Resonance Spectroscopy on a (5-Nanometer) ${ }^{3}$ Sample volume, Science 339 (6119) 561 (2013).

[29] D. Rugar, H. J. Mamin, M. H. Sherwood, M. Kim, C. T. Rettner, K. Ohno, and D. D. Awschalom, Proton magnetic resonance imaging using a nitrogen-vacancy spin sensor, Nat. Nanotechol. 10, 120 (2015).

[30] T. Häberle, D. Schmid-Lorch, F. Reinhard, and J. Wrachtrup, Nanoscale nuclear magnetic imaging with chemical contrast, Nat. Nanotechnol. 10, 125 (2015).

[31] M. Kim, H. J. Mamin, M. H. Sherwood, C. T. Rettner, J. Frommer, and D. Ruger, Ultrafast magnetization switching by spin-orbit torques, Appl. Phys. Lett. 105, 042406 (2014).

[32] M. Kim, H. J. Mamin, M. H. Sherwood, K. Ohno, D. D. Awschalom, and D. Rugar, Decoherence of Near-Surface Nitrogen-Vacancy Centers Due to Electric Field Noise, Phys. Rev. Lett. 115, 087602 (2015).

[33] F. Fávaro de Oliveira, S. Momenzadeh, D. Antonov, H. Fedder, A. Denisenk, and J. Wrachtrup, On the efficiency of combined ion implantation for the creation of nearsurface nitrogen-vacancy centers in diamond, Phys. Status Solidi, 8 2044-2050 (2016).

[34] T. Rosskopf, A. Dussaux, K. Ohashi, M. Loretz, R. Schirhagl, H. Watanabe, S. Shikata, K. M. Itoh, and C. L. Degen, Investigation of Surface Magnetic Noise by Shallow Spins in Diamond, Phys. Rev. Lett. 112, 147602 (2014).

[35] B. A. Myers, A. Das, M. C. Dartiailh, K. Ohno, D. D. Awschalom, and A. C. Bleszynski Jayich, Probing Surface Noise with Depth-Calibrated Spins in Diamond, Phys. Rev. Lett. 113, 027602 (2014).

[36] B. K. Ofori-Okai, S. Pezzagna, K. Chang, M. Loretz, R. Schirhagl, Y. Tao, B. A. Moores, K. Groot-Berning, J. Meijer, and C. L. Degen, Spin properties of very shallow nitrogen vacancy defects in diamond, Phys. Rev. B 86, 081406(R) (2012).

[37] Rogerio De Sousa, Dangling-bond spin relaxation and magnetic $1 / \mathrm{f}$ noise from the amorphoussemiconductor/oxide interface: Theory, Phys. Rev. B 76, 245306 (2007).

[38] N. Bar-Gill, L. Pham, C. Belthangady, D. Le Sage, P. Cappellaro, J. Maze, M. Lukin, A. Yacoby, and R. Walsworth, Suppression of spin-bath dynamics for im- proved coherence of multi-spin-qubit systems, Nat. Commun. 3, 858 (2012).

[39] N. Bar-Gill, L.M. Pham, A. Jarmola, D. Budker, and R. L. Walsworth, Solid-state electronic spin coherence time approaching one second, Nat. Commun. 4, 1743 (2013).

[40] V. Y. Osipov, A. Shames, and A. Y. Vul', Exchange coupled pairs of dangling bond spins as a new type of paramagnetic defects in nanodiamonds, Physica (Amsterdam) 404B, 4522 (2009).

[41] N. D. Samsonenko, G. V. Zhmykhov, V. S. Zon, and V. K. Aksenov, Characteristic features of the electron paramagnetic resonance of the surface centers of diamond, J. Struct. Chem. 20, 951 (1980).

[42] J. Tisler et al., Fluorescence and Spin Properties of Defects in Single Digit Nanodiamonds, ACS Nano 3, 1959 (2009).

[43] L. P. McGuinness et al., Ambient nanoscale sensing with single spins using quantum decoherence, New. J. Phys. 15, 073042 (2013).

[44] R. C. Bansal, F. J. Vastola, and P. L. Walker, Kinetics of chemisorption of oxygen on diamond, Carbon 10, 443 (1972).

[45] H. Bluhm, J. A. Bert, N. C. Koshnick, M. E. Huber, and K. A. Moler, Spinlike Susceptibility of Metallic and Insulating Thin Films at Low Temperature, Phys. Rev. Lett. 103, 026805 (2009).

[46] K. Ohashi, T. Rosskopf, H. Watanabe, M. Loretz, Y. Tao, R. Hauert, S. Tomizawa, T. Ishikawa, J. Ishi-Hayase, S. Shikata, C. L. Degen, and K. M. Itoh, Negatively Charged Nitrogen-Vacancy Centers in a $5 \mathrm{~nm}$ Thin ${ }^{12} \mathrm{C}$ Diamond Film, Nano Lett. 13 (10), 4733 (2013).

[47] K. Ohno, F. Joseph Heremans, L. C. Bassett, B. A. Myers, D. M. Toyli, A. C. Bleszynski Jayich, C. J. Palmstrom, and D. D. Awschalom, Engineering shallow spins in diamond with nitrogen delta-doping, Appl. Phys. Lett. 101, 082413 (2012).

[48] Y. Romach, C. Müller, T. Unden, L. J. Rogers, T. Isoda, K. M. Itoh, M. Markham, A. Stacey, J. Meijer, S. Pezzagna, B. Naydenov, L. P. McGuinness, N. Bar-Gill, and F. Jelezko, Spectroscopy of Surface-Induced Noise Using Shallow Spins in Diamond, Phys. Rev. Lett. 114, 017601 (2015).

[49] H. J. Mamin, M. Kim, M. H. Sherwood, C. T. Rettner, K. Ohno, D. D. Awschalom, and D. Rugar, Nanoscale Nuclear Magnetic Resonance with a Nitrogen-Vacancy Spin Sensor, Science 339 (6119), 557 (2013).

[50] P. Kehayais, A. Jarmola, N. Mosavain, I. Fescenko, F.M. Benito, A. Laraoui, J. Smits, L. Bougas, D. Budker, A. Neumann, S. R. J. Brueck and V. M. Acosta, Solution nuclear magnetic resonance spectroscopy on a nanostructured diamond chip, Nature Communications 8, 188 (2017).

[51] D. R. Glenn, D. B. Bucher, J. Lee, M. D. Lukin, H. Park and R. L. Walsworth, High Resolution Magnetic Resonance Spectroscopy Using Solid-State Spin sensor, Nature volume 555, 351 (2018).

[52] N. Aslam, M. Pfender, P. Neumann, R. Reuter, A. Zappe, F. F. Oliveira, A. Denisko, H. Sumiya, S. Onoda, J. Isoya and J. Wrachtrup, Nanoscale nuclear magnetic resonance with chemical resolution, Science 7, 357, 6346 (2017).

[53] Seppe Kuehn, Roger F. Loring, and John A. Marohn, "Dielectric Fluctuations and the Origins of Noncontact Friction, Phys. Rev. Lett., 96, 156103 (2006).

[54] H.M. Zidan \& M. Abu-Elnader, "Structural and Optical 
Properties of Pure PMMA and Metal Chloride-Doped PMMA Films, Phys. B: Conds. Mat. 355 (1-4), 308-317, (2005).

[55] C. Duan, W.N. Mei, W. Yin, J. Liu, J.R. Hardy, M. Bai, \& S. Ducharme, Theoretical study on the optical properties of polyvinylidene fluoride crysta, J. Conds. Mat. 15 (22), 3805 (2003).

[56] W. Jones Jr., Properties of Perfluoropolyethers for Space Applications, NASA-TM-106616, 19940033009 (1994).

[57] K.A. Akmarov, S.N. Lapshov, \& A.S. Sherstobitova, Optical Properties of Aqueous Solutions of Dimethyl Sulfoxide and Application of Refractometry for Monitoring Their Composition, J. Appl. Spectrosc 80, 610 (2013).

[58] S. Havriliak and S. Negami, A complex plane representation of dielectric and mechanical relaxation processes in some polymers, Polymer 8, 161, 1967.

[59] M. Principe, I. M. Pinto, V. Pierro, R. DeSalvo, I. Taurasi, A. E. Villar, E. D. Black, K. G. Libbrecht, C. Michel, N. Morgado, and L. Pinard, Material loss angles from direct measurements of broadband thermal noise, Phys. Rev. D 91, 022005 (2015).

[60] A. E. Villar, E. D. Black, R. DeSalvo, K. G. Libbrecht, C. Michel, N. Morgado, L. Pinard, I. M. Pinto, V. Pierro, V. Galdi, M. Principe, and I. Taurasi, Measurement of thermal noise in multilayer coatings with optimized layer thickness, Phys. Rev. D 81, 122001 (2010).

[61] G. Harry, A. Gretarsson, P. Saulson, S. Kittelberger, S. Penn, W. Startin, S. Rowan, M. Fejer, D. R. M. Crooks, G. Cagnoli, J. Hough, and N. Nakagawa, Thermal noise in interferometric gravitational wave detectors due to dielectric optical coatings, Class. Quantum Grav. 19, 897 (2002).

[62] G. Harry, H. Armandula, E. Black, D. R. M. Crooks, G. Cagnoli, J. Hough, P. Murray, S. Reid, S. Rowan, P. Sneddon, M. Fejer, R. Route, and S. Penn, Thermal noise from optical coatings in gravitational wave detectors, App. Optics 45, 7, 1569 (2006).

[63] A N Brozdnichenko, D M Dolgintsev, and R A Castro, The dielectric properties of the diamond-like films grown by ion-plasma method, J. Phys.: Conf. Ser. 572, 012025 (2014).

M. Köhler, P. Lunkenheimer, and A. Loidl, Dielectric and conductivity relaxation in mixtures of glycerol with $\mathrm{LiCl}$, Eur. Phys. J. E 27, 115 (2008).

X. You, M. I. Chaudhari, S. B. Rempe, and L. R. Pratt,
Dielectric Relaxation of Ethylene Carbonate and Propylene Carbonate from Molecular Dynamics Simulations, J. Phys. Chem. B, 120 (8), 1849 (2016).

M G Giri, M Carla, C M C Gambi, D Senatra, A Chittofrati and A Sanguineti, Dielectric permittivity measurements on highly conductive perfluoropolyether microemulsions at frequencies up to $100 \mathrm{MHz}$, Meas. Sci. Technol. 4627 (1993).

V. M. Boucher, D. Cangialosi, A. Alegria, J. Colmenero, J. Gonzalez-Irun, and L. M. Liz-Marzan, Physical aging in PMMA/silica nanocomposites: Enthalpy and dielectric relaxation, J. Non-Crys. Sol. 347 (2), 605 (2011).

P. Thomas, K. T. Varughese, K. Dwarakanath, and K. B. R. Varma, Dielectric properties of Poly(vinylidene fluoride) $/ \mathrm{CaCu}_{3} \mathrm{Ti}_{4} \mathrm{O}_{12}$ composites, Comp. Sci. and Tech. 70 (3), 539 (2010).

K. Schröter, S. A. Hutcheson, X. Shi, A. Mandanici, and G. B. Mckenna, Dynamic shear modulus of glycerol: Corrections due to instrument compliance, J. Chem. Phys. 125, 214507 (2006).

A. P. Gregory\& R. N. Clarke, Tables of the complex permittivity of dielectric reference liquids at frequencies up to $5 \mathrm{GHz}$, NPL Report MAT 23 (2012).

[64] A. Safavi-Naini, P. Rabl, P. F. Weck, and H. R. Sadeghpour, Microscopic model of electric-field-noise heating in ion traps, Phys. Rev. A 84, 023412 (2011).

[65] G. S. Agarwal, Quantum electrodynamics in the presence of dielectrics and conductors. I. Electromagnetic-field response functions and black-body fluctuations in finite geometries, Phys. Rev. A 11, 230-242 (1975).

[66] C. Henkel, K. Joulain, J.-P. Mulet, and J.-J. Greffet, Radiation forces on small particles in thermal near fields, J. Opt. A: Pure Appl. Opt. 4, S109-S114 (2002).

[67] J. M. Wylie and J. E. Sipe, Quantum electrodynamics near an interface, Phys. Rev. A 30, 1185 (1984).

[68] M. Constantin, C. C. Yu, and J. M. Martinis, "Saturation of two-level systems and charge noise in Josephson junction qubits, Phys. Rev. B 79, 094520 (2009).

[69] Asma Khalid, Kelvin Chung, Ranjith Rajasekharan, Desmond W.M. Lau, Timothy J. Karle, Brant C. Gibson \& Snjezana Tomljenovic-Hanic, Lifetime Reduction and Enhanced Emission of Single Photon Color Centers in Nanodiamond via Surrounding Refractive Index Modification, Sci. Rep. 5, 11179 (2015). 